\title{
SIGNS SIMULATING THOSE OF MITRAL STENOSIS
}

\author{
BY \\ CRIGHTON BRAMWELL \\ From the Cardiographic Department, Manchester Royal Infirmary \\ Received August 4, 1942
}

Duplication of the second heart sound at the apex, especially when this is associated with a systolic murmur, is apt to be regarded by recruiting medical boards as evidence of an organic mitral lesion. The purpose of this paper is to suggest that this sign is physiological, and to put forward a hypothesis to account for it and for certain other signs that are apt to lead to a mistaken diagnosis of mitral stenosis. In a group of 835 recruits referred to the writer by medical boards of the Ministry of Labour and National Service, a duplicated second heart sound at the apex was heard in 157; this series does not include any case in which an early diastolic murmur would be heard or a presystolic murmur could be elicited by exercise.

The duplicated second sound, like the presystolic murmur of mitral stenosis, is best heard with the patient lying on his left side. As it seemed probable that this was due to the change in the axis of the mitral orifice with change of posture, the writer sought the help of Professor Wood Jones, who kindly examined a series of cadavers hardened in formalin and found that, relative to the long axis of the body, the blood stream issuing through the mitral orifice was directed mainly forwards with an inclination of about $20^{\circ}$ downwards and slightly towards the left. Thus, with the patient lying on his back, the blood stream is flowing almost directly against gravity, whereas when he turns on his left side it is flowing horizontally. The latter position would tend to accelerate the blood flow from auricle to ventricle, and so, as will be shown later, would render conditions more favourable for duplication of the second heart sound.

The second element of the duplicated second heart sound follows the closure of the semilunar valves by about $0 \cdot 1 \mathrm{sec}$. It therefore corresponds in time to the physiological third heart sound; in fact the two appear to be identical. Thayer (1908) found that a third heart sound could be heard in 65 per cent of healthy young persons. He also demonstrated, experimentally in dogs, a similar sound which corresponded in time to the sudden distension of the ventricle that occurs early in diastole, and he suggested that the third heart sound was due to sudden tension of the A-V valves produced by the first rush of blood from auricle to ventricle.

The physiological third heart sound is much more commonly heard in young than in older subjects. The same is true of the duplicated second sound in recruits. Table I gives the age incidence of the 157 cases in the present series as compared with the age incidence of the whole group of 835 cases among which they occurred.

TABLE I

Age Incidence of Duplicated Second Sound

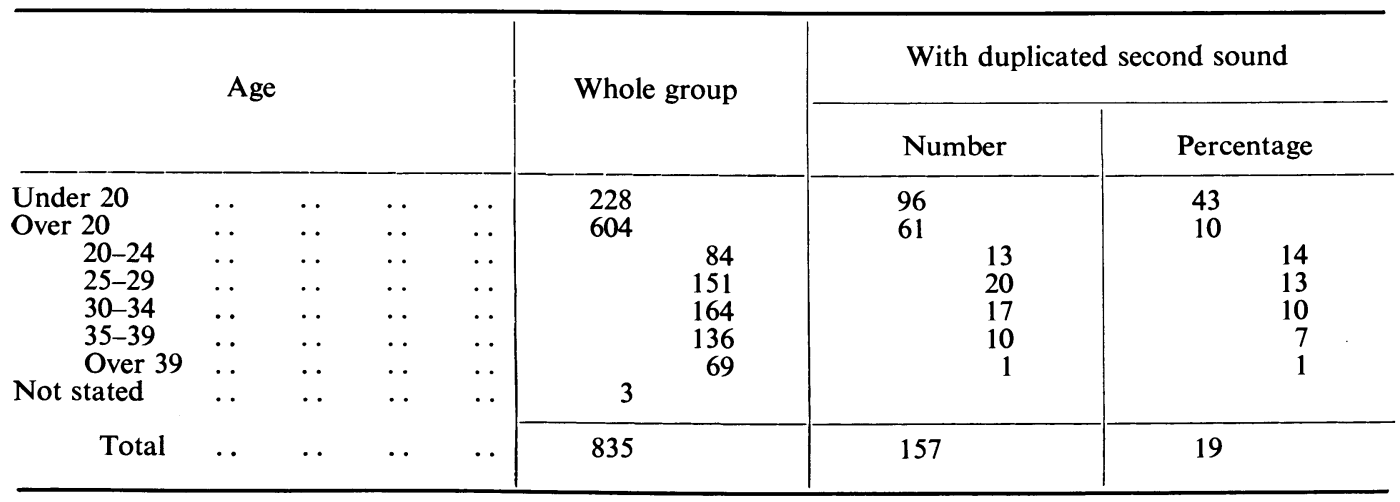


From this table it will be seen that whereas the incidence was 19 per cent in the whole group, it was 43 per cent in men under twenty as compared with only 10 per cent in men over that age.

Most of these young men appeared to be perfectly fit; some were athletic and many were well above average physique. In the opinion of the writer, 70 per cent were fit for Grade I, and of the 46 cases placed in lower categories, 22 were lads of eighteen or nineteen whose physical development was in arrear of their age. They were accordingly temporarily placed in Grade II or III. Thirteen only were down-graded on account of permanently poor physique, and $\mathbf{1 1}$ for other reasons.

So far as pulse rate and systolic blood pressure are concerned there was remarkably little difference between recruits with a duplicated second sound and the entire group of which they formed part. In both cases the mode for the systolic pressure was 130-140. Only 10 per cent of the recruits with a duplicated second sound, however, had a diastolic pressure over 80 as compared with 22 per cent in the entire group. This is not unexpected in view of the lower average age in the former group.

Only a few of the electrocardiograms exhibited right axis deviation, but in most cases radioscopy did suggest that in men with a duplicated second heart sound the pulmonary arc was more prominent than usual, the heart conforming to the childish contour. William Evans (1942), whose observations were based on actual measurments, came to the same conclusion.

In these recruits, the duplicated second sound is generally associated either with a frank apical systolic murmur or with an impure first heart sound; often it was this murmur which was responsible for the case being referred for a second opinion. It seems possible that this murmur is attributable to a safety-valve mitral incompetence, associated with overfilling of the ventricle, and is comparable to the murmur which one hears in normal people immediately after severe physical exertion.

\section{The Presystolic Murmur}

In endeavouring to assess the significance of the duplicated second heart sound and to determine the mode of its production, it is helpful to consider the incidence of the crescendo presystolic murmur of mitral stenosis. In the production of an obstructive murmur two factors are concerned, the size of the orifice and the velocity of the blood flow: the one is static, the other dynamic. No matter how small the orifice, a murmur will only be produced when the blood flowing through it attains a certain critical velocity. The importance of the dynamic factor is well illustrated by following patients with mitral stenosis through pregnancy. In many of these cases, a loud presystolic murmur is heard during the later months of pregnancy: but, after delivery, no murmur is audible at rest, though one can be elicited by exercise. In such cases, it is obvious that the presence or absence of a murmur is determined not by the size of the mitral orifice but by the velocity of the blood flow.

There is an inverse mathematical relation between the degree of stenosis and the velocity of the blood flow required to produce an obstructive murmur. When stenosis is considerable a murmur is heard at rest, but when it is trivial there is no murmur unless the rate of blood flow is increased by exercise. The following observations suggest that even when the mitral orifice is normal, an obstructive murmur may be produced if the rate of blood flow be sufficiently increased.

In the course of a routine examination of athletes at the Olympic Games in Amsterdam, Ellis and the writer (1931) found that, in certain perfectly normal subjects, the first heart sound might be so modified that it was indistinguishable from the presystolic murmur and accentuated first heart sound of mitral stenosis. This sign was present in 12 out of 192 athletes examined; three were Marathon runners, three long-distance runners, and three long-distance cyclists-all athletes whose particular form of sport entailed prolonged and severe exertion. It was not present in any of the 18 sprinters or 16 middle-distance runners included in this series. A similar observation was made by Sewall (1909), twenty years previously. His explanation was that when the ventricle is filled at a certain rate, the reflux of the blood current tends to bring the cusps of the mitral valve into approximation, so that the auricle, in expelling its contents, has to force a channel between them. This explanation is analogous to the Austin Flint hypothesis, but it seems unduly speculative. An alternative explanation is that, in athletes who indulge in prolonged and severe exertion, the auricles share in the general cardiac hypertrophy that is known to occur. Consequently the velocity with which the blood is projected into the ventricles by the hypertrophied auricles is greater than under normal conditions, and the mitral orifice proves relatively too small for the increased rate of blood flow.

Further evidence in support of this hypothesis is derived from the fact that in many cases of acute thyrotoxicosis the first heart sound bears a distinct resemblance to that of mitral stenosis. In 1914 Bridgeman demonstrated in graphic records of the normal first heart sound a series of small vibrations synchronous with auricular systole. These were of such low intensity that they failed to reach the threshold of audibility. Amplification of these presystolic vibrations, sufficient to render them audible, accounts for the roughening of the first heart sound in thyrotoxicosis (Bramwell, 1935). A 
similar modification of the first heart sound is liable to occur in any excitable overacting heart and may lead to a mistaken diagnosis of mitral stenosis. This mistake is not surprising; for, if the hypothesis outlined above be correct, there is no auditory line of demarcation between anatomical stenosis due to a narrowed orifice and physiological stenosis due to an increased rate of blood flow. The one is absolute; the other, relative.

\section{The Early Diastolic Murmur}

To revert to consideration of the significance of the duplicated second heart sound at the apex, the diastolic part of the heart cycle may be divided physiologically into three phases in accordance with the velocity of the blood flow from auricle to ventricle. When the mitral valve opens, the pressure in the auricle is much higher than in the ventricle, and the blood which had been accumulating behind the closed valve during systole rushes in a swirling torrent into the empty ventricle. As soon as the initial difference in pressure between the two chambers has been relieved, the rate of blood flow from auricle to ventricle diminishes and the torrent is converted into a quietly flowing stream. Finally, when the auricle contracts, the rate of blood flow through the mitral orifice is again increased.

In extreme stenosis a rumbling murmur is heard throughout diastole, but in slight stenosis it is only during early diastole and presystole that the velocity of blood flow is sufficient to produce a murmur, the intermediate phase of diastasis being silent.

The fact that, in the lesser degrees of mitral stenosis, a presystolic is more common than an early diastolic murmur suggests that the difference in pressure on the two sides of the mitral orifice is greater during auricular systole than during early diastole. This may be due to the fact that in oldstanding cases of mitral stenosis, left auricular hypertrophy is the rule, and the force with which the blood is ejected by the auricle is correspondingly increased.

The classical signs of slight mitral stenosis are a presystolic murmur with an accentuated first and a duplicated second heart sound. When stenosis is more pronounced the duplicated second sound is followed by a diastolic murmur, the duration of which varies with the degree of stenosis.

The second element of the duplicated second sound in mitral stenosis is called by French cardiologists " le claquement d'ouverture de la mitrale" and ascribed to vibrations of the thickened mitral valve set up by the first rush of blood from auricle to ventricle. In this respect its mode of production is similar to that of the normal third heart sound, but in the one case the sound is attributable to the stenosis of the mitral orifice and the thickening of the mitral cusps, whereas in the other the orifice and valves are normal but the rate of blood flow is increased. The former is the pathological, the latter the physiological, third heart sound.

\section{SUMMARY}

In a consecutive series of 835 recruits, a duplicated second heart sound (generally associated with an apical systolic murmur) was present in 157 cases.

The duplicated second heart sound was best heard when the patient lay on his left side; the reason for this is discussed. Duplication of the second heart sound was much more common in men under 20 than in older recruits. Seventy per cent of the men in whom it was present were considered fit for Grade I. Radioscopy in these cases generally showed an increased prominence of the pulmonary arc.

Since the production of an obstructive murmur depends on the degree of obstruction relative to the velocity of the blood current, it is suggested that an increased rate of blood flow through a normal mitral orifice may be instrumental in producing: $(a)$ the accentuation and roughening of the first heart sound heard in certain athletes, in thyrotoxicosis, and in other conditions in which the heart is overacting, and $(b)$ the duplicated second heart sound heard in healthy subjects. Both these signs are therefore regarded as signifying a "relative" mitral stenosis.

This hypothesis entails a physiological conception of mitral stenosis based on the volume of blood which an orifice of a certain size can transmit in unit time.

\section{REFERENCES}

Bramwell, C. (1935). Quart. J. Med., 4, N.S., 139.

Bramwell, C., and Ellis, R. (1931). Quart. J. Med., 24, 329.

Bridgeman, E. W. (1914). Arch. intern. Med., 13, 475.

Evans, W. (1942). Personal communication.

Sewall, H. (1909). Amer. J. med. Sci., 138, 10.

Thayer, W. S. (1908). Trans. Assoc. Amer. Phys., 23, 326. 